\title{
In vivo studies disprove an obligatory role of azurin in denitrification in Pseudomonas aeruginosa and show that azu expression is under control of RpoS and ANR
}

\author{
Erik Vijgenboom, Julie E. Busch and Gerard W. Canters
}

Leiden Institute of Chemistry, Gorlaeus Laboratories, Leiden University, PO Box 9502, 2300 RA Leiden, The Netherlands Author for correspondence: Erik Vijgenboom. Tel: +31 71 5274278. Fax: +31 715274349.
e-mail : vijgenbo@chem.leidenuniv.nl

\begin{abstract}
The role of the blue copper protein azurin and cytochrome $c_{551}$ as the possible electron donors to nitrite reductase in the dissimilatory nitrate reduction pathway in Pseudomonas aeruginosa have been investigated. It was shown by an in vivo approach with mutant strains of $P$. aeruginosa deficient in one or both of these electron-transfer proteins that cytochrome $c_{551}$, but not azurin, is functional in this pathway. Expression studies demonstrated the presence of azurin in both aerobic and anaerobic cultures. A sharp increase in azurin expression was observed when cultures were shifted from exponential to stationary phase. The stationary-phase sigma factor, $\sigma^{s}$, was shown to be responsible for this induction. In addition, one of the two promoters transcribing the azu gene was regulated by the anaerobic transcriptional regulator ANR. An azurin-deficient mutant was more sensitive to hydrogen peroxide and paraquat than the wild-type $P$. aeruginosa. These results suggest a physiological role of azurin in stress situations like those encountered in the transition to the stationary phase.
\end{abstract}

Keywords: azurin, redox stress, ANR, rpoS, anaerobiosis

\section{INTRODUCTION}

The blue copper protein azurin, a periplasmic redox protein and member of the cupredoxin family (Canters, 1987; Wood, 1978), has been identified in several bacteria such as Alcaligenes xylosoxidans NCTC 8582 (formerly Alcaligenes denitrificans; Hoitink et al., 1990), Methylobacillus flagellatum KT (Gak et al., 1995), Methylomonas J (Ambler \& Tobari, 1989) and Alcaligenes xylosoxidans NCIMB 11015 (Dodd et al., 1995). The first report of azurin was made by Horio (1958), who isolated the protein from Pseudomonas aeruginosa and designated it 'Pseudomonas blue protein'. Subsequent investigations demonstrated that significant amounts of this protein could be isolated from $P$. aeruginosa grown under oxygen limitation (Yamanaka et al., 1961). The finding of azurin expression during anaerobic respiratory growth with nitrate as the final electron acceptor and in vitro experiments carried out over the years has led to the hypothesis that azurin is involved in dissimilatory nitrate reduction (Barber $e$ t al., 1976; Silvestrini et al., 1982; Zannoni, 1989).

Cloning of the azurin gene, azu, from $P$. aeruginosa
(Canters, 1987; Arvidsson et al., 1989) provided the possibility of investigating the mechanism underlying expression regulation and the correlation to specific growth conditions. Mapping of the transcription start sites identified two promoters, of which the proximal promoter (P1) appeared to be the stronger (Arvidsson $e t$ al., 1989). Based on sequence homology two sequence elements which could be of importance in the regulation of transcription initiation were identified in the promoter region. The $\mathrm{P} 2$ promoter region has similarity with the $\sigma^{\mathrm{N}}$ consensus sequence (Arvidsson et al., 1989; Hoitink et al., 1990), although the spacing between the strictly conserved GG and GC motifs (Morett \& Buck, 1989 ) is not correct. RNA polymerases containing $\sigma^{\mathrm{N}}$ transcribe various genes involved in nitrogen metabolism, pilus production and many other functions (for a review see Merrick, 1993). A sequence with strong homology with the consensus FNR-binding site (TTGAT- $\mathrm{N}_{4}$-ATCAA) is present upstream of P1 (Hoitink et al., 1990). The structural and functional analogue of FNR in $P$. aeruginosa is ANR, a transcription regulator which can activate transcription during oxygen limitation (Sawers, 1991; Zimmermann et al., 1991). The denitrification pathway and anaerobic arginine degra- 
Table 1. List of strains and plasmids

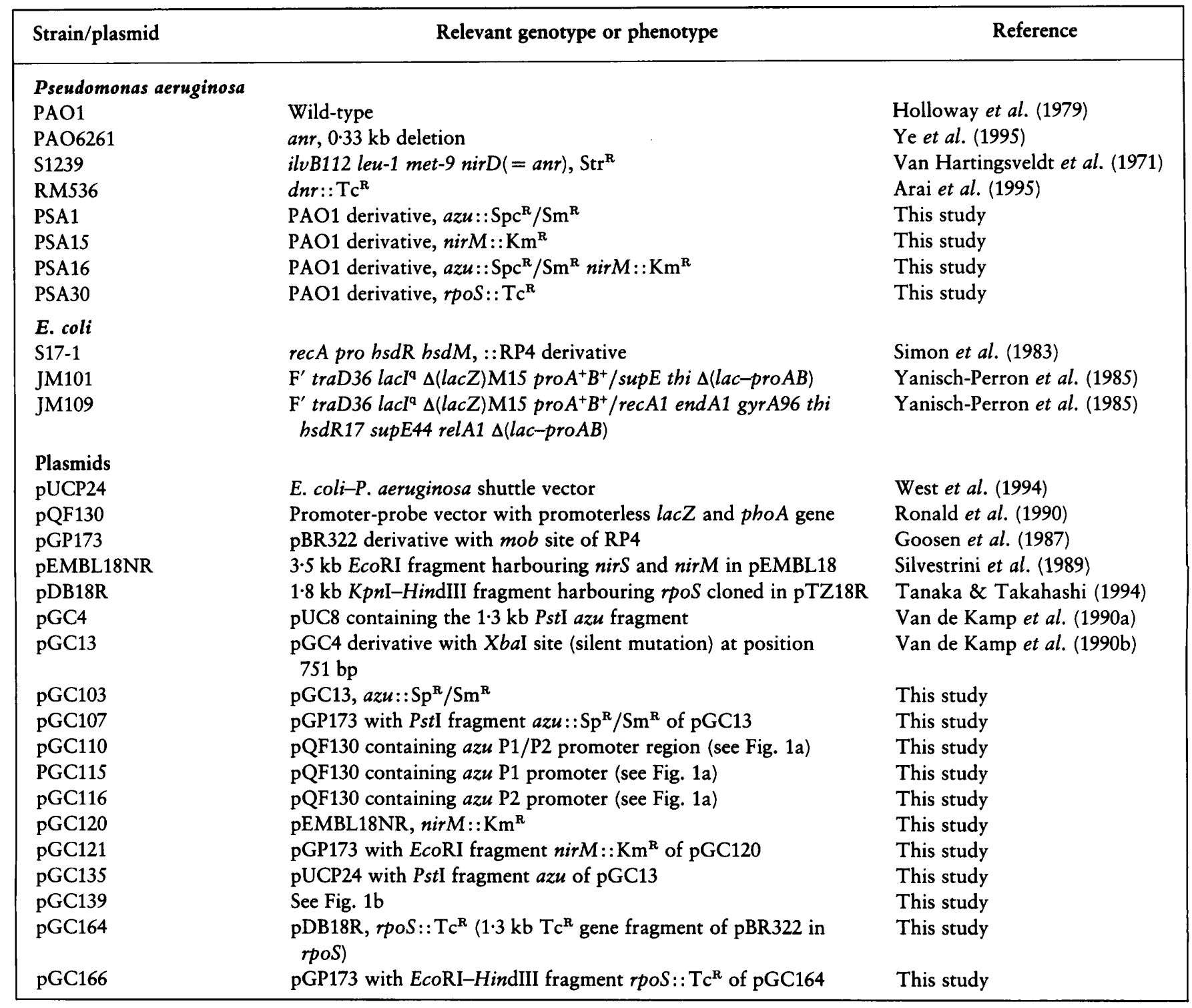

dation are dependent upon ANR for their anaerobic activation in $P$. aeruginosa (Galimand et al., 1991; Ye et al., 1995).

The electron-transport capability of azurin has been well established by in vitro experiments. The purported redox partners of azurin, cytochrome $c_{551}\left(\right.$ cyt $\left.-c_{551}\right)$ and nitrite reductase, have been identified from in vitro electron-transfer studies, but their relevance as physiological partners has not been established in vivo (Horio, 1958; Wilson et al., 1975; Corin et al., 1983; Van de Kamp et al., 1990b; Silvestrini et al., 1994). More recently azurin has also been suggested as the primary electron acceptor for ethanol dehydrogenase in $P$. aeruginosa (Duine, 1995), for aromatic amine dehydrogenase in Alcaligenes faecalis (Edwards et al., 1995; Hyun \& Davidson, 1995), for methylamine dehydrogenase in Methylobacillus flagellatum KT (Gak et al., 1995), for amicyanin in an obligate methylotroph, organism 4025 (Auton \& Anthony, 1989), and as the electron donor to the copper-containing NIR of Pseudomonas aureofaciens (Zumft et al., 1987). Most of these suggestions have arisen from in vitro electron-transfer experiments. Only in the case of Methylobacillus flagellatum has it been shown that an azurin-deficient mutant, although still viable, grew much slower on methylamine than the wild-type strain (Gak et al., 1995).

In this study a detailed investigation of the expression of $a z u$ in several genetic backgrounds under aerobic and anaerobic conditions is presented. These data and the investigation of strains lacking azurin and/or cyt- $c_{551}$ exclude an essential physiological role of azurin in dissimilatory nitrate reduction in $P$. aeruginosa. The sensitivity of the $a z u$ mutant of $P$. aeruginosa towards reactive oxygen species such as hydrogen peroxide and superoxide radicals generated by paraquat point to a function for azurin in redox stress response. 
(a)

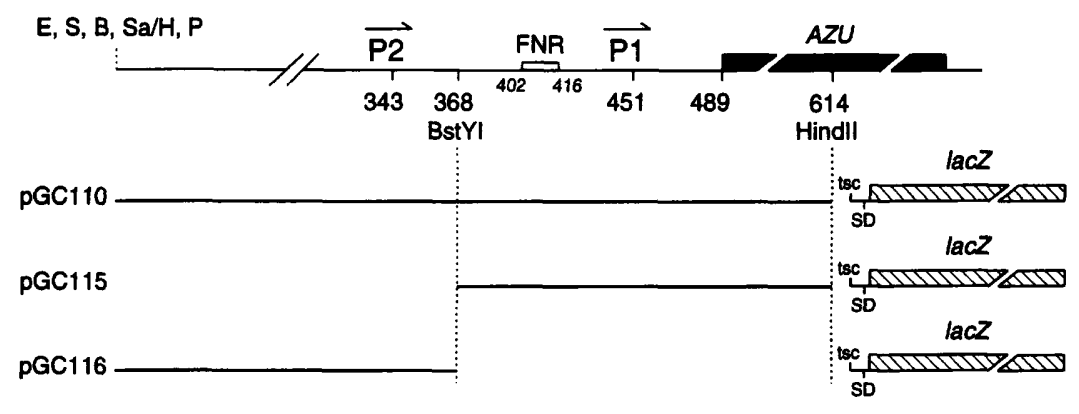

(b)

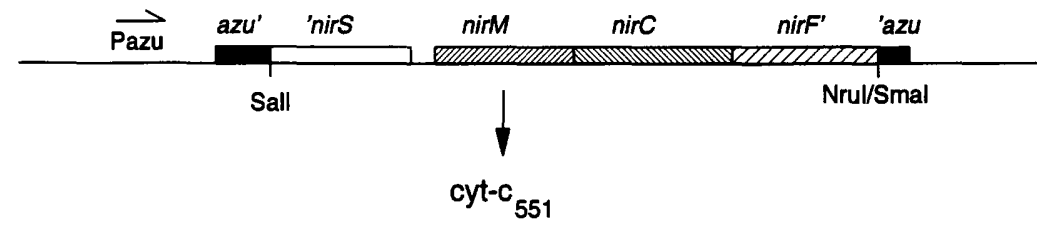

Fig. 1. (a) Promoter-probe constructs in PQF130 (for details see Methods). The complete azu promoter region HindII-HindII (pGC110), P1 BstYl-HindII (pGC115) and P2 Hindll-BstYI (pGC116) were cloned upstream of the lacZ gene of PQF130. The lacZ gene is preceded by a ribosome-binding site (SD) and translational stop codons (tsc). Relevant restriction sites are indicated. The restriction sites in the multiple cloning site of PQF130 are EcoRI (E), Smal (S), BamHI (B), Sall/HindII (Sa/H) and Pstl (P). (b) The Sall-Nrul fragment of PEMBL18NR (Silvestrini et al., 1989) was ligated in Sal//Smal-digested pGC135 (a pUCP24 derivative containing the Pstl azu fragment of pG(13), resulting in pGC139. The promoter region and the first 42 amino acids (including the leader sequence) of azurin are fused in-frame to the C-terminal 107 aa of nitrite reductase (nirs). The construct also includes the complete ORF of nirC, a monohaem cytochrome $c$ of unknown function and the N-terminal part of nirf. The product of nirf is suggested to take part in the biosynthetic pathway of haem $d_{1}$ (Kawasaki et al., 1995).

\section{METHODS}

Strains and media. Strains are listed in Table 1. LB (LuriaBertani) medium (Miller, 1992) and defined minimal medium (MM), VB medium (Vogel \& Bonner, 1956) supplemented with trace-element solution, or phosphate-buffered minimal medium were used for both Escherichia coli and P. aeruginosa cultures. Aerobic growth conditions in liquid cultures were maintained by vigorous shaking of Erlenmeyer flasks filled to a maximum of $10 \%$ of the volume and incubated at $37^{\circ} \mathrm{C}$. Anaerobic conditions were obtained in tightly capped bottles which were filled to $95 \%$ with medium. Anaerobic incubation of agar plates was done at $37^{\circ} \mathrm{C}$ in an Oxoid anaerobic jar containing Oxoid AnaeroGen. Media for anaerobic growth contained $50 \mathrm{mM} \mathrm{KNO}_{3}$ or $10 \mathrm{mM} \mathrm{NaNO}{ }_{2}$. MM contained $0.2 \%$ yeast extract when used under oxygen-limiting conditions. Plasmids were maintained by adding the appropriate antibiotic at the following concentrations: for E. coli, $100 \mu \mathrm{g}$ ampicillin $\mathrm{ml}^{-1}, 50 \mu \mathrm{g}$ streptomycin $\mathrm{ml}^{-1}, 25 \mu \mathrm{g}$ neomycin $\mathrm{ml}^{-1}, 50 \mu \mathrm{g}$ spectinomycin $\mathrm{ml}^{-1}, 20 \mu \mathrm{g}$ tetracycline $\mathrm{ml}^{-1}, 15 \mu \mathrm{g}$ gentamycin $\mathrm{ml}^{-1}$; and for $P$. aeruginosa, $250 \mu \mathrm{g}$ carbenicillin $\mathrm{ml}^{-1}, 200 \mu \mathrm{g}$ streptomycin $\mathrm{ml}^{-1}, 200 \mu \mathrm{g}$ neomycin $\mathrm{ml}^{-1}$, $1000 \mu \mathrm{g}$ spectinomycin $\mathrm{ml}^{-1}, 150 \mu \mathrm{g}$ tetracycline $\mathrm{ml}^{-1}, 200 \mu \mathrm{g}$ gentamycin $\mathrm{ml}^{-1}$.

Plasmid constructions. The plasmids constructed and used in this study are listed in Table 1. To construct the promoterprobe plasmids, the complete $a z u$ promoter region was isolated as a HindII fragment (Fig. 1a) and cloned in the SmaI site of pQF130, resulting in pGC110. The plasmid containing the correct orientation of the fragment with respect to the $l a c Z$ gene of the vector was identified by restriction analysis. The P1 and $\mathrm{P} 2$ promoters could be separated by digestion with Bst $\mathrm{YI}$. The 236 bp Bst YI-HindII fragment contained P1 and was cloned in BglII/SmaI-digested pQF130, resulting in pGC115. The 370 bp SalI-Bst YI fragment contained P2 and was cloned in SalI/BamHI-digested pQF130, resulting in pGC116. The azurin gene on pGC13 was disrupted by inserting the aadA gene (spectinomycin and streptomycin resistance; Prentki \& Krisch, 1984) in the unique $X b a I$ site at position 751 , resulting in pGC103. The $a z u:: \mathrm{Spc}^{\mathrm{R}} / \mathrm{Sm}^{\mathrm{R}}$ PstI fragment was transferred to PstI-digested pGP173 to create pGC107. The nirM gene (cyt- $c_{551}$ ) was disrupted on PEMBL18NR by cloning the $\mathrm{Neo}^{\mathrm{R}}$ cassette of pUC4K (Pharmacia) in the ApaI site in the coding sequence of nirM. The complete nirS-nirM cluster was cloned in pGP173 as an EcoRI fragment, resulting in pGC121. For the disruption of $r p o S$, pGC164 was constructed by inserting the $1.3 \mathrm{~kb}$ EcoRI-StyI Tc ${ }^{\mathrm{R}}$ fragment of pBR322 $\mathrm{H}$ (HindIII site removed by filling in the sticky ends) into HindII/BamHIdigested pDB18R. Non-compatible restriction sites were blunt-ended. The mobilizable plasmid pGC166 was constructed by insertion of the EcoRI-HindIII fragment harbouring $r p o S:: \mathrm{Tc}^{\mathbb{R}}$ in pGP173. The cyt- $c_{551}$ expression plasmid pGC139 was constructed as outlined in the legend to Fig. 1 (b).

Strain constructions. Inactivation of the genomic copies of $a z u$, nirM and $r p o S$ was carried out by introducing into $P$. aeruginos a suicide plasmid harbouring the inactivated gene. Recombination events between homologous sequences on the genome and those on the plasmid resulted in a gene replacement, leaving the inactive gene copy in the genome. All gene inactivations were checked by antibiotic resistance and by Southern hybridization of total DNA with three probes: (1) the gene of interest, (2) the resistance gene and (3) vector sequences. Plasmids were introduced into $P$. aeruginosa by mating with $E$. coli strain S17-1 carrying the plasmid of interest. Strains were grown until mid-exponential phase in LB medium with the appropriate antibiotic(s), mixed in ratios varying from $1: 1$ to $1: 5$ and grown for $16 \mathrm{~h}$ on LB agar plates. Cells were harvested from the plates and selection for the recombinant $P$. aeruginosa was carried out by plating dilutions on MM plates with glucose and the appropriate antibiotic(s). Individual colonies were screened for their antibiotic resistance, grown in LB medium and chromosomal DNA was analysed as described above.

Recombinant DNA technology. Cloning of DNA fragments was carried out according to standard procedures (Sambrook et al., 1989). E. coli strains were transformed by the method of Chung et al. (1989). pQF130 and pUCP24 derivatives were introduced in $P$. aeruginosa by electroporation. Electrocompetent $P$. aeruginosa cells were prepared from an over- 


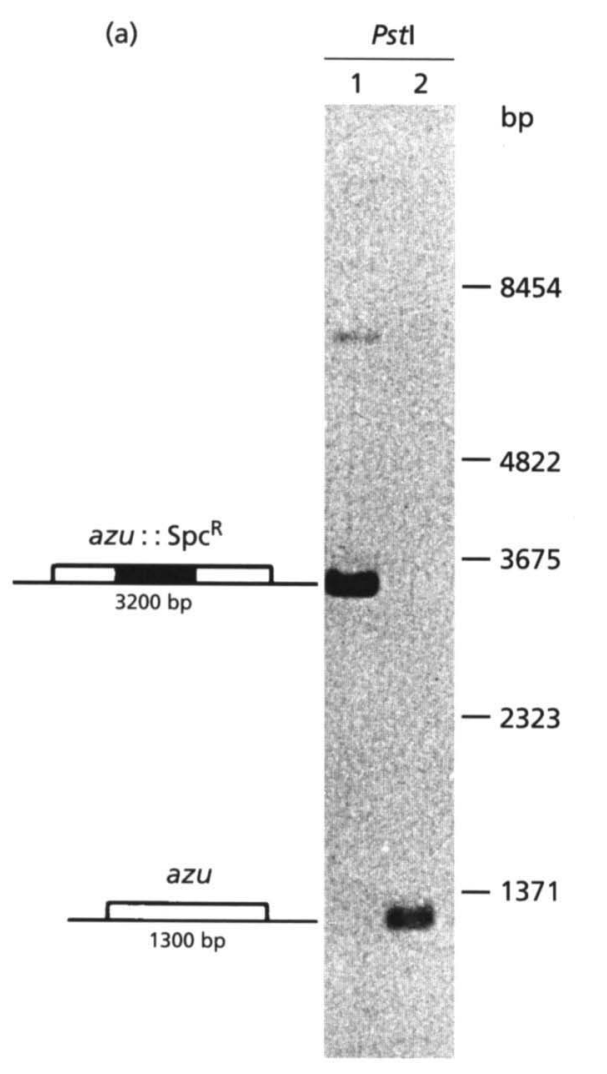

(b)

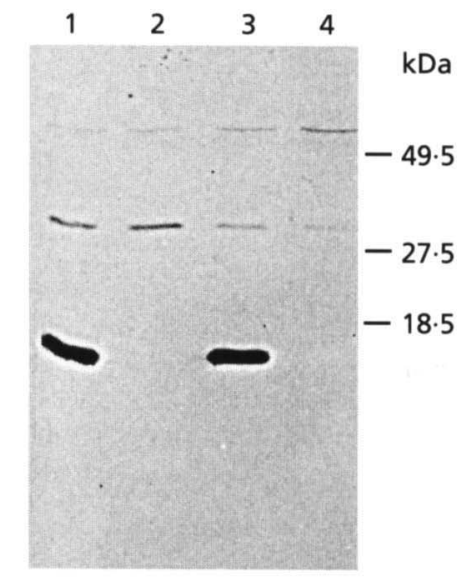

(c)

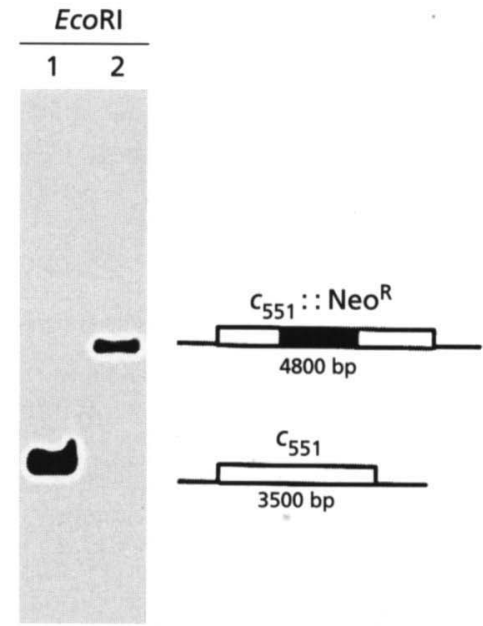

(d)

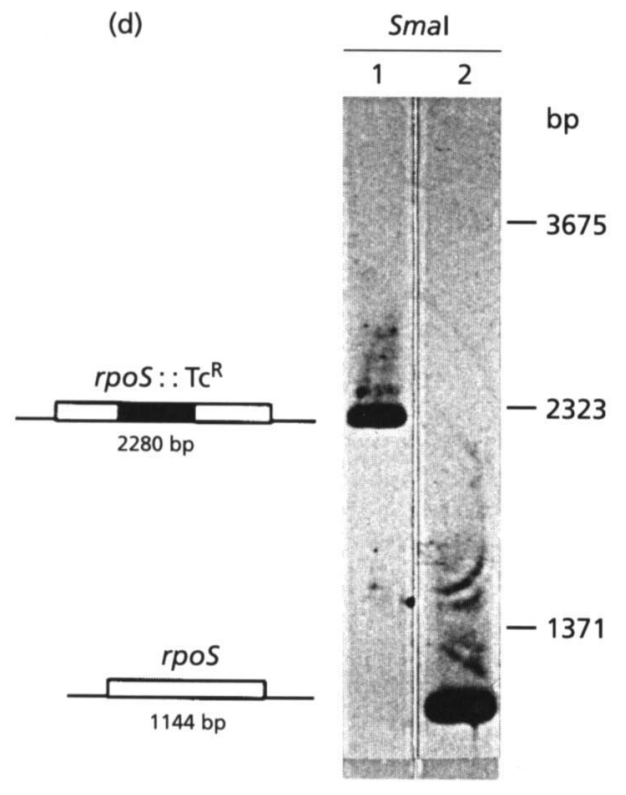

Fig. 2. (a) Southern blot of Pstl-digested total DNA of PSA1, a strain in which the azu gene is disrupted by the insertion of the $\operatorname{aadA}$ gene (lane 1) and of the wild-type strain PAO1 (lane 2). The blot was probed with a randomly labelled (DIG-dUTP) 1300 bp Pstl fragment containing the entire azu gene. On the left-hand side of the blot the expected genomic Pstl fragments are indicated. (b) Western blot with antibodies directed against $P$. aeruginosa azurin. Cells of overnight cultures were pelleted, suspended in SDS loading buffer and samples were run on a $15 \%$ SDS-polyacrylamide gel. Lane 1, PAO1 (wild-type); 2, PSA1 (azu); 3, PSA15 (nirM); 4, PSA16 (azu, nirM). (c) Southern blot of EcoRI-digested total DNA of PAO1 (lane 1) and PSA15 (lane 2) probed with the $1500 \mathrm{bp}$ Sall fragment of pEMBL18NR containing the entire nirM (cyt- $c_{551}$ ) gene. The genomic EcoRI fragment containing nirM is 3500 bp in PAO1 and 4800 bp in PSA15 as a result of gene replacement with nirM::Neo ${ }^{R}$. (d) Southern blot of Smal-digested genomic DNA of PSA30 (lane 1) and PAO1 (lane 2). The Smal fragment carrying the entire rpoS gene is 1144 bp in the wild-type strain. In strain PSA30 the Smal fragment of rpoS migrates more slowly as a result of the gene replacement with rpoS::TCR. night culture in $\mathrm{LB}$ with $10 \mathrm{mM} \mathrm{MgSO}_{4}$ which was diluted 100 -fold in fresh medium and grown at $37^{\circ} \mathrm{C}$ with good aeration for another $5 \mathrm{~h}$. Cells were collected by centrifugation and washed twice with $13 \%$ (v/v) glycerol, $1 \mathrm{mM}$ MOPS ( $\mathrm{pH}$ not adjusted) and the cell pellet was finally suspended in $1 \mathrm{ml}$ of the same buffer. Electroporation was carried out with settings of $1250 \mathrm{~V}, 50 \mu \mathrm{F}$ and $200 \Omega$. Immediately thereafter $1 \mathrm{ml} \mathrm{LB}$ with $10 \mathrm{mM} \mathrm{MgSO}_{4}$ was added and the cells were allowed to recover for $1 \mathrm{~min}$ at room temperature. The antibiotic resistance was expressed at $37^{\circ} \mathrm{C}$ for $60 \mathrm{~min}$ in a total volume of $2.5 \mathrm{ml} \mathrm{LB}$ with $10 \mathrm{mM} \mathrm{MgSO}_{4}$ before plating on LB plates with the appropriate antibiotic. Southern hybridization was done according to standard procedures with capillary transfer of DNA to Hybond- $\mathrm{N}^{+}$(Amersham) or positively charged nylon membrane (Boehringer Mannheim) in $10 \times$ SSC. Hybridization conditions were stringent $(0 \cdot 1 \times$ SSC) and probes were labelled non-radioactively using the DIG system (Boehringer Mannheim). 
Enzyme assay. $\beta$-Galactosidase activity was determined directly in chloroform/SDS-treated cells as described by Miller (1992). Measurements were done in duplicate and cells from three independent cultures were used.

Western blotting. Samples were run on a $15 \%$ SDS-polyacrylamide gel and subsequently blotted to Hybond-C Super (Amersham) in the Bio-Rad Mini-Protean II system. The blot buffer consisted of $12.5 \mathrm{mM}$ Tris, $96 \mathrm{mM}$ glycine $\mathrm{pH} 8.3$ and $20 \%$ methanol. Following the blotting procedure membranes were washed briefly in PBS $(140 \mathrm{mM} \mathrm{NaCl}, 30 \mathrm{mM} \mathrm{KCl}$, $8 \mathrm{mM} \mathrm{Na} \mathrm{HPO}_{4}, 2 \mathrm{mM} \mathrm{KH_{2 }} \mathrm{PO}_{4}, \mathrm{pH} 7.4$ ) and incubated overnight at $4{ }^{\circ} \mathrm{C}$ in $\mathrm{PBS}$ containing $5 \%$ milk (solution prepared from low-fat milk powder) and azurin serum $(1: 2500)$ or nitrite reductase serum $(1: 1000)$. The membranes were then washed three times ( $15 \mathrm{~min}$ per wash) with PBS and incubated for $60 \mathrm{~min}$ at room temperature with TBST (1 $\mathrm{mM}$ Tris/ $\mathrm{HCl}, 150 \mathrm{mM} \mathrm{NaCl}, 0.05 \%$ Tween $20, \mathrm{pH} 7 \cdot 4$ ) containing anti-rabbit IgG alkaline phosphatase conjugate (1: $5000)$. Staining was carried out in AP buffer $(100 \mathrm{mM}$ Tris/ $\mathrm{HCl}, 100 \mathrm{mM} \mathrm{NaCl}, 5 \mathrm{mM} \mathrm{MgCl}, \mathrm{pH} 9.5)$ with NBT and BCIP (Gibco BRL).

\section{RESULTS}

\section{Physiological role of azurin}

To construct a $P$. aeruginosa strain deficient in azurin production we first inactivated the $a z u$ gene on plasmid pGC13 by insertion of the spectinomycin/streptomycinresistance gene $(\operatorname{aad} A)$ in the middle of the coding sequence. The resulting plasmid, pGC103, was used to construct strain PSA1 by gene replacement (Fig. 2a). Western blot analysis using antibodies raised against purified azurin demonstrated that azurin was no longer produced in the mutant strain (Fig. 2b).

The growth characteristics (doubling time, maximum $\mathrm{OD}_{600}$ ) of PSA1 ( $\left.a z u\right)$ grown under aerobic conditions in LB medium were indistinguishable from those of the wild-type strain PAO1. Only upon addition of nitrite $(10 \mathrm{mM})$ was a slightly reduced growth rate observed. Also under anaerobic conditions either in the presence of nitrate or nitrite the strain grew as well as did the wild-type (Table 2). An essential function of azurin in denitrification is therefore excluded. The current model for the electron-transport pathway used by $P$. aeruginosa during anaerobic growth with nitrate or nitrite as the final electron acceptor has both azurin and cyt- $c_{551}$ as electron donors for NIR. To identify the in vivo donor we constructed strains derived from PAO1 that lack either cyt- $c_{551}$ (PSA15) or both azurin and cyt- $c_{551}$ (PSA16). The cyt- $c_{551}$ gene (nirM) was inactivated by gene replacement with a nirM copy disrupted by the neomycin-resistance gene (Fig. 1b). In strains PSA15 and PSA16 the growth rate under anaerobic conditions was reduced by $50 \%$ in medium containing nitrate. Under the same conditions, with nitrite as the final electron acceptor, the generation time increased at least fourfold to $500 \mathrm{~min}$ or more and the maximum $\mathrm{OD}_{600}$ was reduced (Table 2). Disruption of the nirM gene (cyt- $c_{551}$ ) might have a negative influence on the expression of the upstream nirS (nitrite reductase) gene. However, in both strains the expression of NIR as judged by Western blotting with antibodies directed against NIR was comparable to that in PAO1, as was the case for the incorporation of haem in NIR (data not shown). In accordance with this observation, the anaerobic growth on nitrate or nitrite of both PSA15 and PSA16 could be restored to almost wild-type performance by complementation of the genomic cyt- $c_{551}$ mutation with pGC139, a plasmid harbouring and expressing nirM but not nirS. Therefore, strains PSA15 and PSA16 do produce an active nitrite reductase. All of this demonstrates that cyt- $c_{551}$ is a physiological electron donor of NIR in $P$. aeruginosa.

Two other options for the physiological function of azurin could be excluded by analysing the growth of PSA1 and PAO1 on media with ethanol or tyramine as the sole carbon source. Both carbon sources supported growth of the wild-type strain PAO1 and the azu mutant PSA1 to a comparable level. It is, therefore, highly unlikely that azurin is the obligatory electron acceptor for ethanol dehydrogenase or aromatic amine dehydrogenase in PAO1.

\section{Control of azurin expression}

Since azurin is not involved in the physiological processes described above, we turned to expression studies of azu in $P$. aeruginosa to obtain a clue as to its physiological role. The temporal expression of $a z u$,

Table 2. Growth characteristics of wild-type and mutant $P$. aeruginosa

\begin{tabular}{|c|c|c|c|c|c|c|c|c|}
\hline \multirow[t]{2}{*}{ Strain } & \multicolumn{4}{|c|}{$\mathrm{LB}+50 \mathrm{mM} \mathrm{KNO}$} & \multicolumn{4}{|c|}{$\mathrm{LB}+10 \mathrm{mM} \mathrm{NaNO}$} \\
\hline & $+\mathrm{O}_{2}^{*}$ & $-\mathrm{O}_{2}{ }^{*}$ & Gast & $\mathrm{OD}_{600} \neq$ & $+\mathrm{O}_{2}^{*}$ & $-\mathrm{O}_{2}{ }^{*}$ & Gast & $\mathrm{OD}_{600} \neq$ \\
\hline PAO1 (wild-type) & 33 & 65 & ++ & $1 \cdot 2$ & 40 & 120 & ++ & 0.55 \\
\hline PSA1 $\left(\mathrm{Azu}^{-}\right)$ & 33 & 65 & ++ & $1 \cdot 2$ & 45 & 130 & ++ & 0.50 \\
\hline PSA15 $\left(\right.$ Cyt $\left.-c_{551}{ }^{-}\right)$ & 35 & 90 & + & 0.8 & 40 & 540 & - & $0 \cdot 18$ \\
\hline PSA16 $\left(\mathrm{Azu}^{-}, \mathrm{Cyt}-\mathrm{C}_{551}{ }^{-}\right)$ & 35 & 90 & + & 0.6 & 45 & 570 & - & $0 \cdot 15$ \\
\hline
\end{tabular}

* Doubling times $( \pm 3 \mathrm{~min}$ ) are the mean values from three independent cultures.

† A rough indication of the gas production $\left(\mathrm{N}_{2}, \mathrm{~N}_{2} \mathrm{O}\right)$ in the anaerobic cultures is given as judged by the amount of gas bubbles in the bottles.

$\ddagger$ The maximum $\mathrm{OD}_{600}$ of the anaerobic cultures is indicated. 
(a)

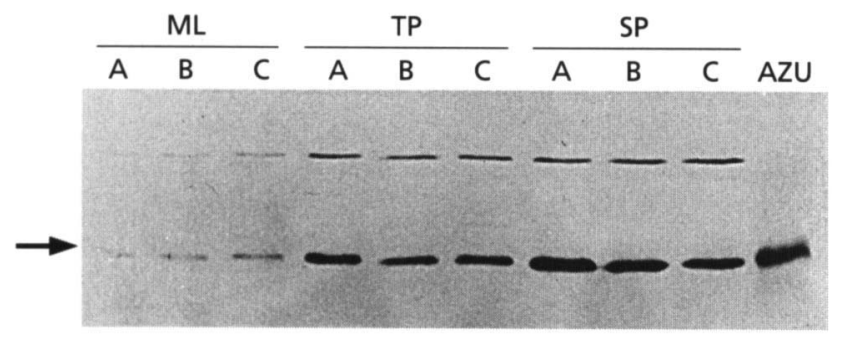

(b)

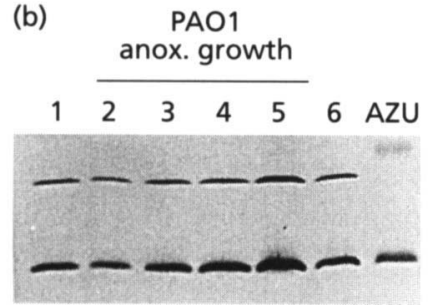

Fig. 3. (a) Western blot of whole-cell extracts of PAO1 (A), PAO6261 (B) and 51239 (C). Strains were grown under aerobic conditions $(10 \mathrm{ml}$ LB medium in a $100 \mathrm{ml}$ Erlenmeyer flask with a cotton plug and incubated in a New Brunswick model G25 incubator shaker at 250 r.p.m.) in LB medium at $37^{\circ} \mathrm{C}$. Samples were taken at an $O D_{600}$ (as determined by a Shimadzu UV-2101 PC UV-VIS scanning electrophotometer) of 0.4 (ML, midexponential-phase samples; lanes 1-3), 1.5 (TP, transition-phase samples; lanes 4-6) and 4.0 (SP, stationary-phase samples; lanes 7-9) and run on a $15 \%$ SDS-polyacrylamide gel. Lane 10 , purified azurin (AZU) $50 \mathrm{ng}$. The position of azurin is indicated by the arrow. (b) Western blot of whole-cell extracts of PAO1. Lanes 1 and 6, grown aerobically for $16 \mathrm{~h}$ in LB. Lanes 2-5, grown anaerobically in $\mathrm{LB}$ with $50 \mathrm{mM} \mathrm{KNO}_{3}$. Lane 2, exponential phase; lane 3, late-exponential phase; lane 4, transition phase; lane 5, stationary phase. The last lane contains a sample of purified azurin showing a faint band in the upper part of the gel which is due to azurin dimers. All samples were normalized for the same amount of cells. The upper band in lanes 1-9 in (a) and lanes 1-6 in (b) is a cross-reaction of the antibodies and is probably not related to azurin since it was also observed in extracts of the azurin-deficient strain PSA1 (see Fig. 2b).

under a variety of conditions, was analysed by Western blotting of whole-cell extracts. The results of samples taken at three representative time-points, mid-exponential phase $(\mathrm{ML})$, transition phase $(\mathrm{TP})$ and stationary phase (SP), from batch cultures growing with good aeration is shown in Fig. 3(a). In the wild-type strain
PAO1 (lanes A), little azurin was present in the cell during exponential growth. However, upon transition to the stationary phase, a significant amount of azurin was detected. The high level of azurin expression continued into the stationary phase with little change between samples from the early and late stationary phase. In the anr mutants PAO6261 and S1239 (Fig. 3a, lanes $B$ and $C$ respectively) the azurin concentration also increased in the transition phase but the final amount in the stationary phase was lower than that observed in PAO1. The lower azurin content in stationary-phase cells (i.e. low oxygen pressure in the medium) of the two anr mutants and the presence of an ANR-binding site in the azu promoter region is in agreement with a regulatory role for ANR in azurin expression.

Control of azurin expression by ANR predicts a higher expression level under anaerobic growth conditions. This is shown in Fig. 3 (b) for the wild-type strain. Both the basal level of expression, i.e. the amount of azurin observed during exponential growth, and the final level in the stationary phase were higher during anaerobic growth than were the levels observed in an aerobic culture. An increase in anaerobic expression was observed during the transition to stationary phase, indicating that a second regulatory mechanism is in operation. The increase in azurin levels observed in the transition from the exponential to the stationary phase under aerobic conditions may be due to the concerted action of ANR and this second regulatory mechanism.

\section{Promoter activity in PAO1 under aerobic and anaerobic conditions}

Upstream of the $a z u$ gene two promoters have been identified by $\mathrm{S} 1$ mapping (Arvidsson et al., 1989). One of them, $\mathrm{P} 1$, is preceded by a sequence, TTGAC- $\mathrm{N}_{4}^{-}$ ATCAG, with high similarity to the consensus recognition site of FNR (identical bases are underlined). The distal promoter, P2, has the GG and GC motifs characteristic for promoters recognized by RNA polymerase containing the alternative $\sigma^{\mathrm{N}}$ factor, although the motifs do not have the correct spacing (Fig. 4). The activity of both promoters was assayed in strains harbouring lacZ promoter-probe constructs (Fig. 1a). Total activity was determined with plasmid pGC110 containing both $\mathrm{P} 1$ and $\mathrm{P} 2$. The individual contributions of the promoters were measured with the constructs pGC115 (P1) and pGC116 (P2).

\section{P2 AAGCGTTGCCGGACGTTOCOTCGCAGGCGCGAAGCGGCACATCTGTGCTAAAACAGGAGT 344}

\section{P1 GGGTTTGACCTGAATCAGTGGAACTCGGTGCCCAATCGGGCAGTCTGCTCTTTCAGAATT 458}

$$
-35
$$$$
-10
$$

Fig. 4. Nucleotides are numbered according to Hoitink et al. (1990). Transcription start sites are at $T(344)$ and $T(458)$. From the data in the paper by Arvidsson et al. (1989) it is difficult to determine the start site of the P1 promoter. The authors indicate position T(457), but the present alignment on the basis of sequence homology shows that T(458) is more likely. The top sequence is the region upstream of the P2 start site and the bottom sequence is upstream of the P1 start site. Identical bases are in bold face. The 'FNR-binding sequence' upstream of the -35 region of P1 is underlined. The GG and GC motifs in the $\mathrm{P2}$ promoter region are in italics and underlined. 


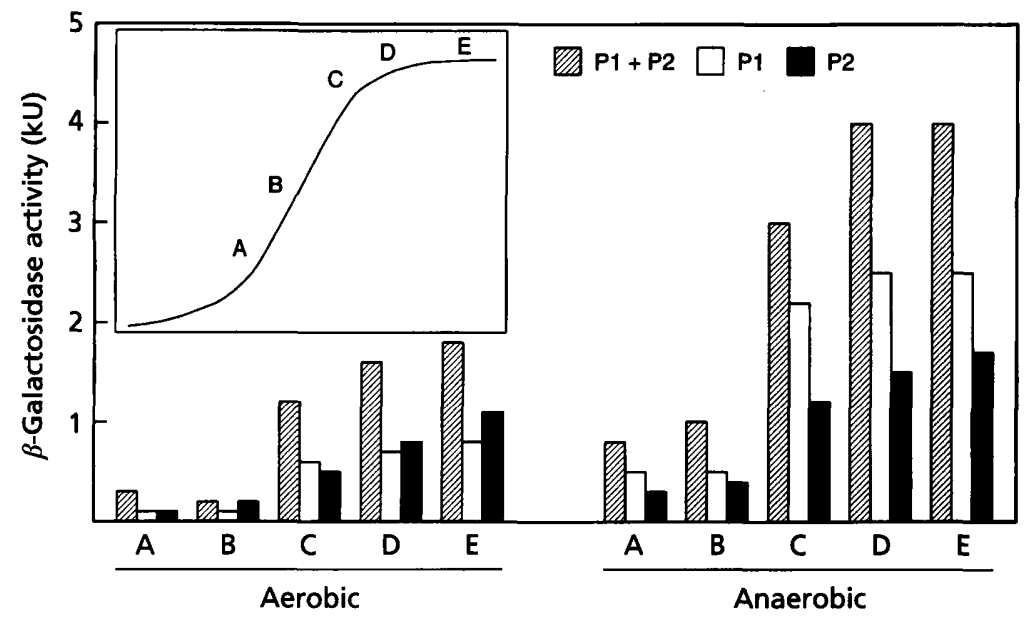

Fig. 5. The activities of the azurin promoters are expressed in $\beta$-galactosidase units (kU) and were determined in wild-type $P$. aeruginosa PAO1. The promoter-probe plasmids, pGC110 (P1 + P2), pGC115 (P1) and pGC116 (P2), contain the azurin promoter(s) upstream of lacZ (see Methods). Cells were grown in LB medium (aerobic) or LB medium with $50 \mathrm{mM} \mathrm{KNO}$ (anaerobic). Samples were taken at the points (A, B, C, D and $E$ ) indicated in the graph of the growth curve (inset).

\section{Table 3. Promoter activities}

Promoter activities are expressed as $\beta$-galactosidase activities (in thousands of Miller units, $\mathrm{kU}$ ) in cells in the exponential phase (exp) and stationary phase (stat). Aerobic cultures were grown in LB medium (10 ml per $100 \mathrm{ml}$ flask) with vigorous shaking. Anaerobic cultures were grown in LB medium with $50 \mathrm{mM} \mathrm{KNO}_{3}$ in tightly capped bottles filled to $95 \%$ volume. Data are corrected for differences in plasmid copy number. The plasmid copy number in an exponential culture of PAO1 harbouring pGC110 is used as a reference. The relative copy numbers were determined by counting the number of transformants obtained in $E$. coli after transformation with plasmid DNA isolated from an equal amount of cells from each of the P. aeruginosa cultures used in this study. Little fluctuation has been observed in the copy number of the various constructs. The $\beta$-galactosidase values represent the mean of at least three independent experiments. The individual measurements differed by $15 \%$ or less. Under all conditions the sum of the measurements of the individual promoters is equal to the value measured for the intact promoter region. Therefore, it is highly unlikely that separation of the two promoters affects the regulation of the individual promoters.

\begin{tabular}{|c|c|c|c|c|c|c|}
\hline \multirow[t]{2}{*}{ Growth conditions } & \multicolumn{2}{|c|}{ PAO1 } & \multicolumn{2}{|c|}{ PAO6261 } & \multicolumn{2}{|c|}{ PSA30 } \\
\hline & $\exp$ & stat & $\exp$ & stat & $\exp$ & stat \\
\hline \multicolumn{7}{|l|}{ Aerobic } \\
\hline pGC110 (P1 + P2) & $0 \cdot 2$ & $1 \cdot 8$ & $0 \cdot 3$ & $1 \cdot 4$ & $0 \cdot 3$ & 0.8 \\
\hline pGC115 (P1) & $0 \cdot 1$ & $0 \cdot 8$ & $0 \cdot 1$ & $0 \cdot 5$ & $0 \cdot 1$ & 0.3 \\
\hline pGC116 (P2) & $0 \cdot 2$ & $1 \cdot 1$ & $0 \cdot 2$ & $1 \cdot 1$ & $0 \cdot 2$ & 0.5 \\
\hline \multicolumn{7}{|l|}{ Anaerobic } \\
\hline pGC110 (P1 + P2) & $1 \cdot 0$ & $4 \cdot 0$ & $0 \cdot 4$ & $1 \cdot 5$ & 0.9 & 1.5 \\
\hline pGC115 (P1) & 0.5 & $2 \cdot 5$ & $0 \cdot 1$ & $0 \cdot 5$ & 0.5 & $1 \cdot 1$ \\
\hline pGC116 (P2) & 0.4 & $1 \cdot 7$ & $0 \cdot 2$ & $1 \cdot 1$ & $0 \cdot 4$ & 0.5 \\
\hline
\end{tabular}

In the wild-type strain PAO1 the activity of both promoters in aerobic cultures was strongly stimulated in the transition phase from exponential growth to stationary phase and the highest activity was observed in stationary-phase cells (Fig. 5). Under anaerobic conditions the promoter activity was higher than under aerobic conditions and there was also an increase in activity with the age of the culture. The P1 activity increased more than did the P2 activity (Fig. 5). The higher promoter activity in the exponential growth phase of an anaerobic culture compared to that of an aerobic culture is an indication of transcription induction by anaerobiosis. On the other hand, the increase in promoter activity during the transition from exponential growth to the stationary phase in an anaerobic culture is a measure of induction by a transcription factor related to stationary-phase growth.

\section{The P1 promoter is regulated by ANR}

The promoter activities were also determined throughout growth in anr and $r p o S$ mutant strains. In all strains promoter activity was low during exponential growth and higher in the stationary phase (Table 3). In the anr mutant PAO6261 the profile of P1 and P2 activity during aerobic growth was similar to that shown for PAO1 in 
Fig. 5. However, the $\mathrm{P} 1$ promoter activity in the stationary phase was reduced by almost $40 \%$ compared to PAO1 (Table 3). Inoculation of PAO6261 under anaerobic conditions in liquid medium resulted in very slow growth, as can be expected from a strain deficient in one of the major transcription factors involved in anaerobiosis. In accordance with the ANR-binding site upstream of $\mathrm{P} 1$, which suggests regulation of $\mathrm{P} 1$ by ANR, the P1 activity in PAO6261 did not respond to anaerobiosis (Table 3). The P2 activity, which increased slightly in PAO1 grown under anaerobic conditions, did not respond to anoxic conditions in PAO6261. Although this promoter does not contain a ANR-binding site, we conclude that the absence of ANR has an effect on its activity. This ANR effect could be indirect and therefore we investigated the promoter activity in a strain deficient in DNR (RM536). This transcription regulator, also belonging to the FNR family, plays a role in switching on the expression of several denitrification genes in response to oxygen starvation (Arai et al., 1995). DNR regulates transcription units including the nirS operon (NIR), the regulatory gene nirQ, and genes of the nitric oxide reductase cluster, norCB. Recently it was determined that $d n r$ is under control of ANR (H. Arai, personal communication). However, promoter activities in RM536 were similar to those in the wild-type strain PAO1 (data not shown), indicating that regulation of P2 activity by DNR is unlikely.

\section{The azu promoters are under control of $\sigma^{5}$}

As mentioned before, a shift to (semi-)anaerobic growth conditions is not the only factor responsible for the increase in $a z u$ expression. The cessation of growth, which takes place during transition to the stationary growth phase could be the other factor. Therefore, the possibility that transcription initiated from P1 and P2 is carried out by an RNA polymerase containing the stationary-phase-specific sigma factor, $\sigma^{\mathrm{s}}$, was investigated. The rpoS gene of $P$. aeruginosa, encoding $\sigma^{\mathrm{s}}$, was recently cloned and sequenced by Tanaka $\&$ Takahashi (1994). The expression of $r p o S$ is controlled according to the growth phase and similar to that of $a z u$. In the exponential growth phase the expression of rpoS is low, but during transition to stationary-phase transcription it is strongly induced (Fujita et al., 1994). The activities of the two azu promoters were measured in PSA30, a rpoS mutant constructed by gene replacement which disrupted the coding region of rpoS by insertion of the tetracycline-resistance gene. In stationary-phase cells of aerobic and anaerobic cultures the activity of both promoters was reduced by more than $50 \%$ compared to the wild-type strain PAO1 (Table 3). Western-blot analysis of PAO1 and PSA30 samples confirmed the reduction of azurin expression in PSA30. Activation of P1 by ANR was still fully operational in the rpoS mutant (compare P1 activity in aerobic cultures with that in anaerobic cultures in Table 3 ). The fact that azurin is still expressed in PSA30 indicates that RNA polymerase containing other $\sigma$ factors can take over in the absence of $\sigma^{\mathrm{s}}$.
Table 4. Hydrogen peroxide and paraquat sensitivity of wild-type and $\mathrm{Azu}^{-} \mathrm{P}$. aeruginosa

Strains were grown in LB at $37^{\circ} \mathrm{C}$ and plated on LB agar plates. Whatman AA discs ( $7 \mathrm{~mm}$ diameter) were impregnated with $10 \mu \mathrm{l}$ of $30 \% \mathrm{H}_{2} \mathrm{O}_{2}$ or $10 \mu \mathrm{l}$ of $100 \mathrm{mM}$ paraquat and positioned in the middle of the agar plate. Following an incubation for $16 \mathrm{~h}$ at $37^{\circ} \mathrm{C}$ the inhibition zones (diameter in $\mathrm{mm}$ ) were measured. Values are the means $( \pm 1 \mathrm{~mm})$ of two experiments.

\begin{tabular}{|lccc|}
\hline Strain & Relevant phenotype & $\mathrm{H}_{2} \mathrm{O}_{2}$ & Paraquat \\
\hline PAO1 & Wild-type & 20 & 18 \\
PSA1 & $\mathrm{Azu}^{-}$ & 29 & 25 \\
PSA1(pGC135) & $\mathrm{Azu}^{-} / \mathrm{Azu}^{+}$ & 21 & 19 \\
\hline
\end{tabular}

\section{Azurin is involved in the response to redox stress}

The enhancement of azurin expression at the onset of and during the stationary phase indicates that the physiological role of azurin may be found in redox processes related to changes in growth conditions. The transition of a bacterial culture to the stationary phase is dependent on many factors such as nutrient limitation, reduced oxygen pressure and the presence of toxic metabolic end-products. We decided to test whether the response to stress conditions in $P$. aeruginosa depends on the presence of azurin. The wild-type strain PAO1 and the azurin-mutant PSA1 were compared for their responses to heat and cold shock, nutritional shiftdown, and for their growth on various carbon sources. However, in none of these experiments was a significant difference between PAO1 and PSA1 observed. Only upon challenging both strains with hydrogen peroxide and the superoxide generator paraquat did a significant difference show. The growth-inhibition zones around the disks were taken as a measure of the sensitivity of the strains to the chemicals applied to the disks. This assay clearly demonstrated that the $a z u$ mutant was more sensitive to both compounds (Table 4). The twofold increase in sensitivity of PSA 1 was also confirmed on gradient plates and in liquid cultures containing paraquat or hydrogen peroxide. To exclude polar effects of the mutation in PSA1, the strain was transformed with pGC135, harbouring and expressing $a z u$, and it was demonstrated that the sensitivity to both paraquat and hydrogen peroxide was restored to wild-type levels (Table 4). The hypothesis that azurin operates in electron transfer mechanisms related to the cellular response to redox stress will be investigated further in future experiments.

\section{DISCUSSION}

Azurin expression analysis by Western blotting showed that production is induced by two distinct growth conditions, the transition from exponential phase to stationary phase and anaerobiosis. It is concluded from these measurements that the trigger for enhanced azurin 
production must be related to the cessation of growth and the limitation of oxygen in the culture. Investigation of the regulation of the two $a z u$ promoters showed that the activity of both promoters is dependent on the presence of sigma factor $\sigma^{\mathrm{s}}$. In the absence of RNA polymerase containing $\sigma^{\mathrm{S}}\left(\mathrm{E} \sigma^{\mathrm{S}}\right)$ the promoter activity is more than $50 \%$ reduced. Despite the low homology between the two promoter regions (Fig. 4), both P1 and P2 seem to be under the control of $\sigma^{\mathrm{s}}$. It is tempting to conclude that both promoters are recognized by $\mathrm{E} \sigma^{\mathrm{s}}$, but we cannot exclude the possibility that a transcription factor whose expression is regulated by $\mathrm{E} \sigma^{\mathrm{S}}$ is involved. In addition to control by $\sigma^{\mathrm{s}}$, the $\mathrm{P} 1$ promoter is also regulated by ANR, a transcription factor which triggers the expression of genes involved in anaerobiosis (Sawers, 1991; Zimmermann et al., 1991; Winteler \& Haas, 1996). Although the $P 2$ promoter region has similarity to promoters recognized by RNA polymerase containing the alternative $\sigma^{\mathrm{N}}$ factor, we could not find an effect of $\mathrm{NH}_{4} \mathrm{Cl}, \mathrm{KNO}_{3}$ or $\mathrm{NaNO}_{2}$ on the overall azu expression or the individual promoter activities (data not shown). Therefore, a regulatory role for these compounds or $\sigma^{\mathrm{N}}$ is not likely.

The gene disruption experiments demonstrate that azurin is not an obligatory redox partner of NIR and that azurin does not take over the electron donation from cyt- $c_{551}$ under anaerobic conditions with nitrite as the final electron acceptor. Surprisingly, the cyt- $c_{551}$ mutants PSA15 and PSA16 did grow in liquid anaerobic cultures with nitrate, although slower and to a lower final cell density than did the wild-type strain (Table 2). Zennaro et al. (1993) have shown that a nirS disruption mutant of $P$. aeruginosa no longer grows anaerobically with nitrate as the acceptor, and De Boer et al. (1994) observed in Paracoccus denitrificans that all nir mutants were severely reduced in anaerobic growth with $\mathrm{KNO}_{3}$. We therefore conclude that in PSA15 and PSA16 either a low level of nitrite, produced by the nitrate reductase, is tolerated, or an alternative and less efficient electrontransport pathway to NIR may be operational.

None of the functions of azurin put forward in the literature (Duine, 1995; Edwards et al., 1995; Hyun \& Davidson, 1995) could be confirmed in vivo. The azu mutant did grow on ethanol like the wild-type strain, albeit slowly, and the primary aromatic amine tyramine did serve as a carbon source for both PAO1 and PSA1. Two possibilities that could not be tested because the corresponding electron-transfer pathways are not known to be present in $P$. aeruginosa are a role for azurin as the electron donor to a copper-containing NIR (Zumft et al., 1987) and as the electron acceptor of methylamine dehydrogenase (Gak et al., 1995). In the light of the observations we described for $P$. aeruginosa it is unlikely that azurin is needed as the obligatory component in any of the corresponding pathways.

In vitro data show that azurin is capable of interacting with various redox proteins, but the in vivo data suggest that these interactions are not of importance under physiological conditions. The question therefore remains as to the physiological function of azurin. The expression profile of azurin points in the direction of an electron-transfer function during times of stress, such as in transition to the stationary phase. Challenging the wild-type strain PAO1 and the azu-mutant PSA1 with hydrogen peroxide and the oxygen radical generator paraquat in a diffusion assay revealed a much higher sensitivity of the mutant strain. We therefore propose that the physiological role of azurin involves electron transfer directly related to the cellular response to redox stress. The need for an increased expression of azurin in the exponential phase of anaerobically growing cells could be related for instance to the observation made in $E$. coli that nitric oxide induces the oxidative stress response (Nunoshiba et al., 1993). In P. aeruginosa nitric oxide is produced by nitrite reductase and, to avoid intracellular damage caused by nitric oxide which is not immediately converted to nitrous oxide by nitric oxide reductase, a stress response could be triggered with a concomitant higher expression of azurin.

The cytochrome $c$ peroxidase (CCP) of $P$. aeruginosa has been shown to interact with azurin in vitro (Foote $e t$ al., 1992). Thus, CCP might be one of the physiological partners of azurin. The recent cloning of the CCP gene from P. aeruginosa (Ridout et al., 1995) can be of help in future in vivo and in vitro investigations to identify the true redox partner(s) of azurin.

\section{ACKNOWLEDGEMENTS}

We are grateful to the late Professor M. C. Silvestrini for the gift of nitrite reductase serum, and we thank Drs D. Haas and H. Arai for the P. aeruginosa strains, and Drs K. Tanaka and $\mathrm{H}$. Takahashi for the rpoS clone. Critical reading of the manuscript by Drs C. Dennison and $M$. Ph. Verbeet is gratefully acknowledged.

\section{REFERENCES}

Ambler, R. P. \& Tobari, J. (1989). Two distinct azurins function in the electron-transport chain of the obligate methylotroph Methylomonas J. Biochem J 261, 495-499.

Arai, H., Igarashi, Y. \& Kodama, T. (1995). Expression of the nir and nor genes for denitrification of Pseudomonas aeruginosa requires a novel CRP/FNR-related transcriptional regulator, DNR, in addition to ANR. FEBS Lett 371, 73-76.

Arvidsson, R. H. A., Nordling, M. \& Lundberg, L. G. (1989). The azurin gene from Pseudomonas aeruginosa. Cloning and characterization. Eur J Biochem 179, 195-200.

Auton, K. A. \& Anthony, C. (1989). The role of cytochromes and blue copper proteins in growth of an obligate methylotroph on methanol and methylamine. J Gen Microbiol 135, 1923-1931.

Barber, D., Parr, S. R. \& Greenwood, C. (1976). Some spectral and steady-state kinetic properties of Pseudomonas cytochrome oxidase. Biochem J 157, 431-438.

Canters, G. W. (1987). The azurin gene from Pseudomonas aeruginosa codes for a pre-protein with a signal peptide. FEBS Lett 212, 168-172.

Chung, C. T., Niemela, S. L. \& Miller, R. H. (1989). One-step preparation of competent Escherichia coli: transformation and storage of bacterial cells in the same solution. Proc Natl Acad Sci USA 86, 2172-2175.

Corin, A. F., Bersohn, R. \& Cole, P. E. (1983). pH dependence of the 
reduction-oxidation reaction of azurin with cytochrome $c_{551}$ : role of histidine-35 of azurin in electron transfer. Biochemistry 22, 2032-2038.

De Boer, A. P. N., Reijnders, W. N. M., Kuenen, J. G., Stouthamer, A. H. \& Van Spanning, R. J. M. (1994). Isolation, sequencing and mutational analysis of a gene cluster involved in nitrite reduction in Paracoccus denitrificans. Antonie van Leeuwenboek 66, 111-127.

Dodd, F. E., Hasnain, S. S., Hunter, W. N., Abraham, Z. H. L., Debenham, M., Kanzler, H., Eldridge, M., Eady, R. R., Ambler, R. P. \& Smith, B. E. (1995). Evidence for two distinct azurins in Alcaligenes xylosoxidans (NCIMB 11015): potential electron donors to nitrite reductase. Biochemistry 34, 10180-10186.

Duine, J. A. (1995). Electron transfer from bacterial dehydrogenases. In From Neural Networks and Biomolecular Engineering to Bioelectronics, pp. 87-94. Edited by C. Nicolini. New York: Plenum.

Edwards, S. L., Davidson, V. L., Hyun, Y.-L. \& Wingfield, P. T. (1995). Spectroscopic evidence for a common electron transfer pathway for two tryptophan tryptophylquinone enzymes. J Biol Chem 270, 4293-4298.

Fujita, M., Tanaka, K., Takahashi, H. \& Anemura, A. (1994). Transcription of the principal sigma-factor genes, $r p o D$ and $r p o S$, in Pseudomonas aeruginosa is controlled according to the growth phase. Mol Microbiol 13, 1071-1077.

Foote, N., Turner, R., Brittain, T. \& Greenwood, C. (1992). A quantitative model for the mechanism of action of cytochrome $c$ peroxidase of Ps. aeruginosa. Biochem J 283, 839-843.

Gak, E. R., Chistoserdov, A. Y. \& Lidstrom, M. E. (1995). Cloning, sequencing and mutation of a gene for azurin in Metbylobacillus flagellatum KT. J Bacteriol 177, 4575-4578.

Galimand, M., Gamper, M., Zimmermann, A. \& Haas, D. (1991). Positive FNR-like control of anaerobic arginine degradation and nitrate respiration in Pseudomonas aeruginosa. J Bacteriol 173, 1598-1606.

Goosen, N., Vermaas, D. A. M. \& van de Putte, P. (1987). Cloning of the genes involved in synthesis of coenzyme pyrrolo-quinolinequinone from Acinetobacter calcoaceticus. J Bacteriol 169, 303-307.

Hoitink, C. W. G., Woudt, L. P., Turenhout, J. C. M., Van de Kamp, M. \& Canters, G. W. (1990). Isolation and sequencing of the Alcaligenes denitrificans azurin encoding gene: comparison with the genes encoding blue copper proteins from Pseudomonas aeruginosa and Alcaligenes faecalis. Gene 90, 15-20.

Holloway, B. W., Krishnapillai, V. \& Morgan, A. F. (1979). Chromosomal genetics of Pseudomonas. Microbiol Rev 43, 73-102.

Horio, T. (1958). Terminal oxidation system in bacteria. J Biochem 45, 195-205.

Hyun, Y.-L. \& Davidson, V. L. (1995). Electron transfer reaction between aromatic amine dehydrogenase and azurin. Biochemistry 34, 12249-12254.

Kawasaki, S., Arai, H., Igarashi, Y. \& Kodama, T. (1995). Sequencing and characterisation of the downstream region of the genes encoding nitrite reductase and cytochrome $c_{551}$ (nirSM) from Pseudomonas aeruginosa: identification of the gene necessary for biosynthesis of heme d1. Gene 167, 87-91.

Merrick, M. J. (1993). In a class of its own - the RNA polymerase sigma factor $\sigma^{54}\left(\sigma^{\mathrm{N}}\right)$. Mol Microbiol 10, 903-909.

Miller, J. H. (1992). A Short Course in Bacterial Genetics. Cold Spring Harbor, NY: Cold Spring Harbor Laboratory.

Morett, E. \& Buck, M. (1989). In vivo studies on the interaction of
RNA polymerase- $\sigma^{54}$ with the Klebsiella pneumoniae and Rhizobium meliloti nifH promoters. J Mol Biol 210, 65-77.

Nunoshiba, T., deRojas-Walker, T., Wishnok, J. S., Tannenbaum, S. R. \& Demple, B. (1993). Activation by nitric oxide of an oxidative stress response that defends Escherichia coli against activated macrophages. Proc Natl Acad Sci USA 90, 9993-9997.

Prentki, P. \& Krisch, H. M. (1984). In vitro insertional mutagenesis with a selectable DNA fragment. Gene 29, 303-313.

Ridout, C. J., James, R. \& Greenwood, C. (1995). Nucleotide sequence encoding the di-haem cytochrome $c_{551}$ peroxidase from Pseudomonas aeruginosa. FEBS Lett 365, 152-154.

Ronald, S. L., Kropinski, A. M. \& Farinha, M. A. (1990). Construction of broad-host-range vectors for the selection of divergent promoters. Gene $90,145-148$.

Sambrook, J., Fritsch, E. F. \& Maniatis, T. (1989). Molecular Cloning : a Laboratory Manual. Cold Spring Harbor, NY: Cold Spring Harbor Laboratory.

Sawers, R. G. (1991). Identification and molecular characterization of a transcriptional regulator from Pseudomonas aeruginosa PAO1 exhibiting structural and functional similarity to the FNR protein of Escherichia coli. Mol Microbiol 5, 1469-1481.

Silvestrini, M. C., Tordi, M. G., Colosimo, A., Antonini, E. \& Brunori, M. (1982). The kinetics of electron transfer between Pseudomonas aeruginosa cytochrome $c_{551}$ and its oxidase. Biochem J 203, 445-451.

Silvestrini, M. C., Galeotti, C. L., Gervais, M., Schinina, E., Barra, D., Bossa, F. \& Brunori, M. (1989). Nitrite reductase from Pseudomonas aeruginosa: sequence of the gene and protein. FEBS Lett 254, 33-38.

Silvestrini, M. C., Falcinelli, S., Ciabatti, I., Cutruzzola, F. \& Brunori, M. (1994). Pseudomonas aeruginosa nitrite reductase (or cytochrome oxidase): an overview. Biochimie 76, 641-654.

Simon, R., Priefer, U. \& Puhler, A. (1983). A broad host range mobilisation system for in vivo genetic engineering: transposon mutagenesis in Gram-negative bacteria. Bio/Technology 1, 784 791.

Tanaka, K. \& Takahashi, H. (1994). Cloning, analysis and expression of an rpoS homologue gene from Pseudomonas aeruginosa PAO1. Gene 150, 81-85.

Van de Kamp, M., Hali, F. C., Rosato, N., Finazzi-Agro, A. \& Canters, G. W. (1990a). Purification and characterization of a non-reconstitutable azurin, obtained by heterologous expression of the Pseudomonas aeruginosa azu gene in Escherichia coli. Biochim Biophys Acta 1019, 283-292.

Van de Kamp, M., Silvestrini, M. C., Brunori, M., Van Beeumen, J., Hali, F. C. \& Canters, G. W. (1990b). Involvement of the hydrophobic patch of azurin in the electron-transfer reactions with cytochrome $c_{551}$ and nitrite reductase. Eur J Biochem 194, 109-118.

Van Hartingsveldt, J., Marinus, M. G. \& Stouthamer, A. H. (1971). Mutants of Pseudomonas aeruginosa blocked in nitrate and nitrite dissimilation. Genetics 67, 469-482.

Vogel, H. J. \& Bonner, D. M. (1956). Acetylornithinase of Escherichia coli: partial purification and some properties. $J$ Biol Chem 218, 97-106.

West, S. E., Schweizer, H. P., Dall, C., Sauple, A. K. \& RunyenJanecky, L. J. (1994). Construction of improved Escherichia-Pseudomonas shuttle vectors derived from pUC18/19 and sequence of the region required for their replication in Pseudomonas aeruginosa. Gene 128, 81-86.

Wilson, M., Greenwood, C., Brunori, M. \& Antonini, E. (1975). 
Electron transfer between azurin and cytochrome $c_{551}$ from Pseudomonas aeruginosa. Biochem J 145, 449-457.

Winteler, H. V. \& Haas, D. (1996). The homologous regulators ANR of Pseudomonas aeruginosa and FNR of Escherichia coli have overlapping but distinct specificities for anaerobically inducible promoters. Microbiology 142, 685-693.

Wood, P. M. (1978). Periplasmic location of the terminal reductase in nitrite respiration. FEBS Lett 92, 214-218.

Yamanaka, T., Ota, A. \& Okunuki, K. (1961). A nitrite reducing system reconstructed with purified cytochrome components of Pseudomonas aeruginosa. Biochim Biophys Acta 53, 294-308.

Yanisch-Perron, C., Vieira, J. \& Messing, J. (1985). Improved M13 phage cloning vectors and host strains: nucleotide sequences of the M13mp18 and pUC19 vectors. Gene 33, 103-119.

Ye, R. W., Haas, D., Ka, J.-O., Krishnapillai, V., Zimmermann, A., Baird, C. \& Tiedje, J. M. (1995). Anaerobic activation of the entire denitrification pathway in Pseudomonas aeruginosa requires Anr, an analog of Fnr. J Bacteriol 177, 3606-3609.
Zannoni, D. (1989). The respiratory chains of pathogenic pseudomonads. Biochim Biophys Acta 975, 299-316.

Zennaro, E., Ciabatti, I., Cutruzzola, F., D'Alessandro, R. \& Silvestrini, M. C. (1993). The nitrite reductase gene of Pseudomonas aeruginosa: effect of growth conditions on the expression and construction of a mutant by gene disruption. FEMS Microbiol Lett 109, 243-250.

Zimmermann, A., Reimmann, C., Galimand, M. \& Haas, D. (1991). Anaerobic growth and cyanide synthesis of Pseudomonas aeruginosa depend on anr, a regulatory gene homologous with fnr of Escherichia coli. Mol Microbiol 5, 1483-1490.

Zumft, W. G., Gotzmann, D. J. \& Kroneck, P. M. H. (1987). Type 1, blue copper proteins constitute a respiratory nitrite-reducing system in Pseudomonas aureofaciens. Eur $J$ Biochem 168, 301-307.

Received 19 March 1997; revised 6 June 1997; accepted 10 June 1997. 